\title{
Supplemental data for X-ray analysis
}

\section{$\underline{X-r a y}$ crystal structures of compounds $\mathbf{1}, \mathbf{2}, \mathbf{4 a}$ and 12 .}

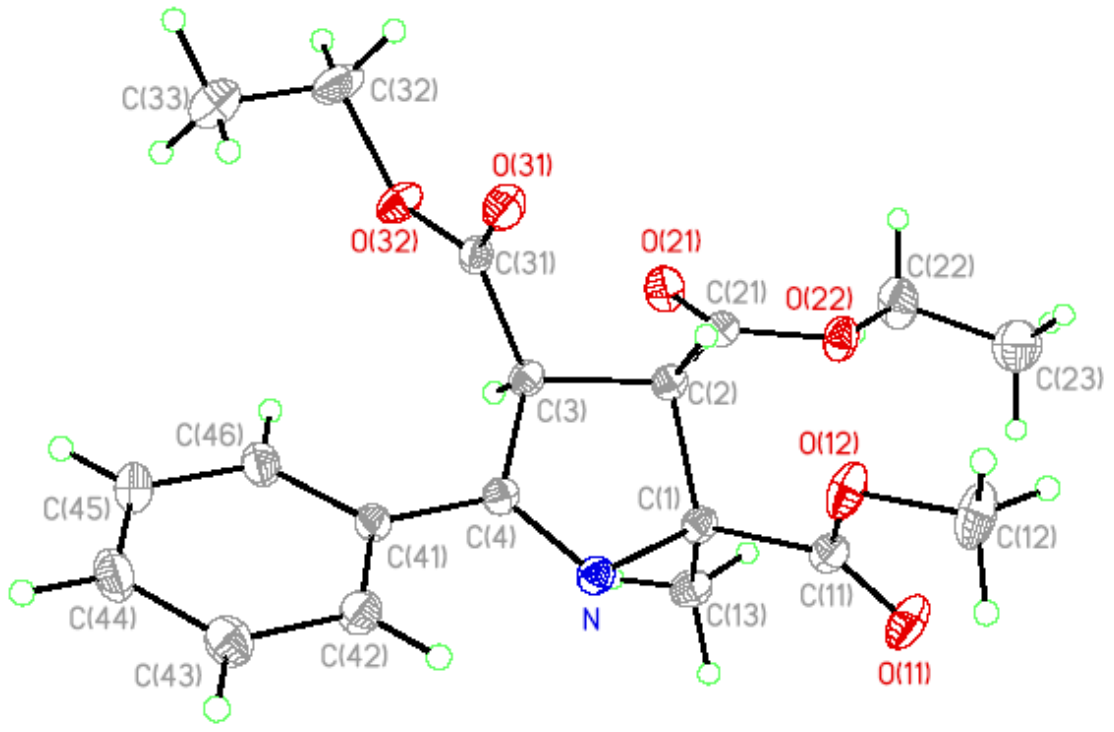

Table 1. Crystal data and structure refinement for 2/4a (crystallized from ether).

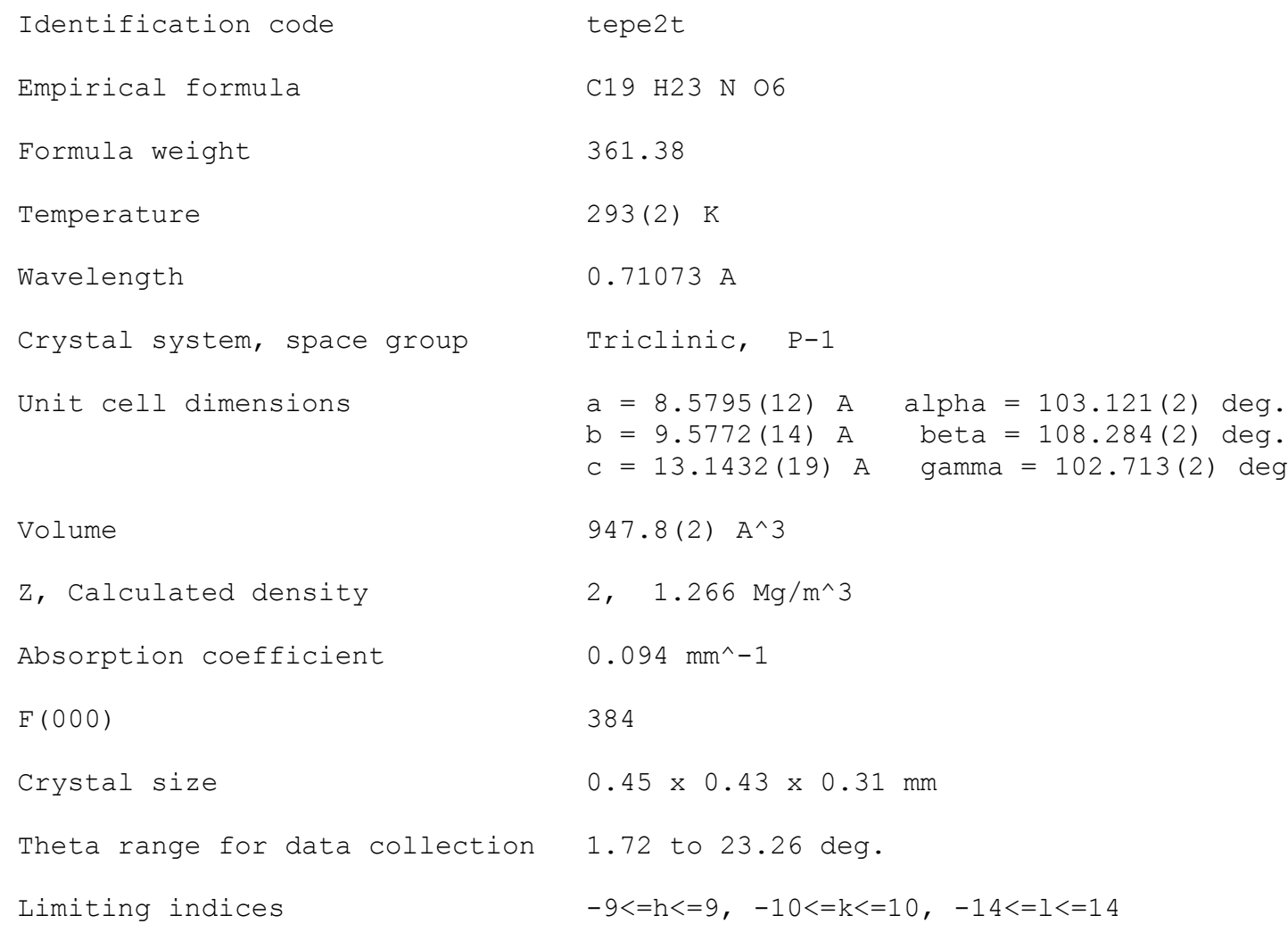




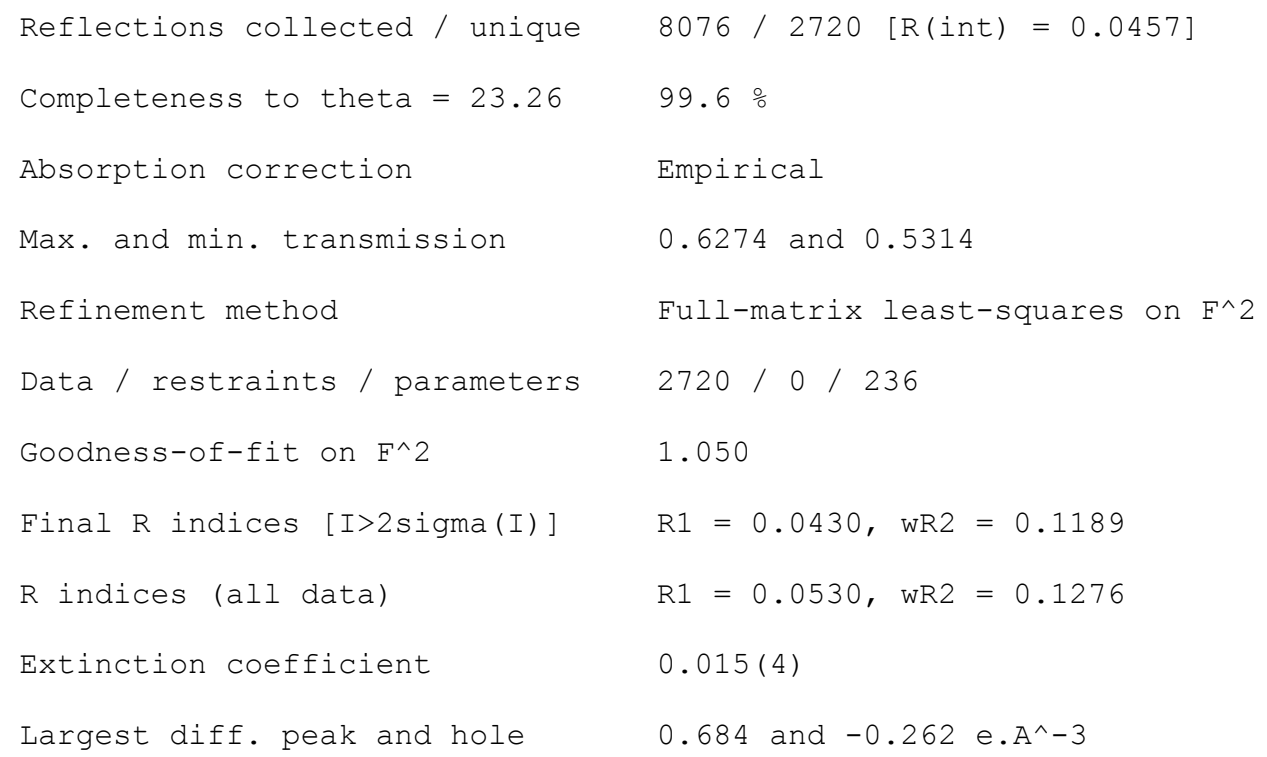

Table 2. Atomic coordinates $\left(x 0^{\wedge} 4\right)$ and equivalent isotropic displacement parameters $\left(A^{\wedge} 2 \times 10^{\wedge} 3\right)$ for $2 / 4 a$.

$\mathrm{U}(\mathrm{eq})$ is defined as one third of the trace of the orthogonalized Uij tensor.

\begin{tabular}{|c|c|c|c|c|}
\hline & $\mathrm{x}$ & $\mathrm{y}$ & z & $\mathrm{U}(\mathrm{eq})$ \\
\hline $\mathrm{N}$ & $-888(2)$ & $-3650(2)$ & $2468(1)$ & $31(1)$ \\
\hline$O(11)$ & $-4076(2)$ & $-6061(2)$ & $2781(2)$ & $54(1)$ \\
\hline$O(12)$ & $-4508(2)$ & $-5141(2)$ & $1338(1)$ & $44(1)$ \\
\hline$O(21)$ & $-2601(2)$ & $-346(2)$ & $4570(1)$ & $45(1)$ \\
\hline$O(22)$ & $-4711(2)$ & $-2585(2)$ & $3724(1)$ & $41(1)$ \\
\hline$O(31)$ & $-2261(2)$ & $-448(2)$ & $1347(1)$ & $42(1)$ \\
\hline$O(32)$ & $-461(2)$ & $1384(2)$ & $2966(1)$ & $43(1)$ \\
\hline$C(1)$ & $-2134(3)$ & $-3635(2)$ & $3036(2)$ & $30(1)$ \\
\hline$C(2)$ & $-2623(3)$ & $-2176(2)$ & $2957(2)$ & $28(1)$ \\
\hline$C(3)$ & $-965(3)$ & $-1130(2)$ & $2986(2)$ & $29(1)$ \\
\hline$C(4)$ & $-245(3)$ & $-2311(2)$ & $2461(2)$ & $29(1)$ \\
\hline C (11) & $-3678(3)$ & $-5082(2)$ & $2398(2)$ & $33(1)$ \\
\hline$C(12)$ & $-5957(3)$ & $-6493(3)$ & $638(2)$ & $55(1)$ \\
\hline$C(13)$ & $-1240(3)$ & $-3577(3)$ & $4256(2)$ & $39(1)$ \\
\hline C (21) & $-3275(3)$ & $-1560(2)$ & $3843(2)$ & $32(1)$ \\
\hline$C(22)$ & $-5458(4)$ & $-2231(3)$ & $4567(2)$ & $54(1)$ \\
\hline$C(23)$ & $-6713(4)$ & $-3607(4)$ & $4471(3)$ & $71(1)$ \\
\hline$C(31)$ & $-1316(3)$ & $-54(2)$ & $2326(2)$ & $30(1)$ \\
\hline C (32) & $-634(4)$ & $2532(3)$ & $2410(2)$ & $55(1)$ \\
\hline C (33) & $633(4)$ & $2759(3)$ & $1878(3)$ & $68(1)$ \\
\hline$C(41)$ & $1100(3)$ & $-1948(2)$ & $1997(2)$ & $30(1)$ \\
\hline$C(42)$ & $1283(3)$ & $-3077(3)$ & $1208(2)$ & $38(1)$ \\
\hline$C(43)$ & $2529(3)$ & $-2742(3)$ & $764(2)$ & $46(1)$ \\
\hline$C(44)$ & $3611(3)$ & $-1277(3)$ & $1103(2)$ & $49(1)$ \\
\hline$C(45)$ & $3467(3)$ & $-148(3)$ & $1897(2)$ & $48(1)$ \\
\hline$C(46)$ & $2209(3)$ & $-486(3)$ & $2340(2)$ & $40(1)$ \\
\hline
\end{tabular}


Table 3. Bond lengths [A] and angles [deg] for 2/4a.

\begin{tabular}{|c|c|}
\hline $\mathrm{N}-\mathrm{C}(4)$ & $1.283(3)$ \\
\hline $\mathrm{N}-\mathrm{C}(1)$ & $1.484(3)$ \\
\hline $\mathrm{O}(11)-\mathrm{C}(11)$ & $1.196(3)$ \\
\hline $\mathrm{O}(12)-\mathrm{C}(11)$ & $1.332(3)$ \\
\hline $\mathrm{O}(12)-\mathrm{C}(12)$ & $1.450(3)$ \\
\hline$O(21)-C(21)$ & $1.205(3)$ \\
\hline $\mathrm{O}(22)-\mathrm{C}(21)$ & $1.336(3)$ \\
\hline$O(22)-C(22)$ & $1.457(3)$ \\
\hline$O(31)-C(31)$ & $1.202(3)$ \\
\hline $\mathrm{O}(32)-\mathrm{C}(31)$ & $1.336(3)$ \\
\hline $\mathrm{O}(32)-\mathrm{C}(32)$ & $1.460(3)$ \\
\hline$C(1)-C(13)$ & $1.527(3)$ \\
\hline$C(1)-C(11)$ & $1.531(3)$ \\
\hline$C(1)-C(2)$ & $1.561(3)$ \\
\hline$C(2)-C(21)$ & $1.503(3)$ \\
\hline$C(2)-C(3)$ & $1.533(3)$ \\
\hline$C(3)-C(31)$ & $1.512(3)$ \\
\hline$C(3)-C(4)$ & $1.535(3)$ \\
\hline$C(4)-C(41)$ & $1.478(3)$ \\
\hline$C(22)-C(23)$ & $1.458(4)$ \\
\hline$C(32)-C(33)$ & $1.470(4)$ \\
\hline$C(41)-C(46)$ & $1.388(3)$ \\
\hline$C(41)-C(42)$ & $1.391(3)$ \\
\hline$C(42)-C(43)$ & $1.383(3)$ \\
\hline$C(43)-C(44)$ & $1.383(4)$ \\
\hline$C(44)-C(45)$ & $1.378(4)$ \\
\hline$C(45)-C(46)$ & $1.391(3)$ \\
\hline $\mathrm{C}(4)-\mathrm{N}-\mathrm{C}(1)$ & $109.27(16)$ \\
\hline$C(11)-O(12)-C(12)$ & $116.20(18)$ \\
\hline$C(21)-O(22)-C(22)$ & $117.31(17)$ \\
\hline$C(31)-O(32)-C(32)$ & $116.91(18)$ \\
\hline $\mathrm{N}-\mathrm{C}(1)-\mathrm{C}(13)$ & $108.88(17)$ \\
\hline $\mathrm{N}-\mathrm{C}(1)-\mathrm{C}(11)$ & $108.33(16)$ \\
\hline$C(13)-C(1)-C(11)$ & $110.14(17)$ \\
\hline $\mathrm{N}-\mathrm{C}(1)-\mathrm{C}(2)$ & $103.65(15)$ \\
\hline$C(13)-C(1)-C(2)$ & $112.51(17)$ \\
\hline$C(11)-C(1)-C(2)$ & $112.99(17)$ \\
\hline$C(21)-C(2)-C(3)$ & $115.87(17)$ \\
\hline$C(21)-C(2)-C(1)$ & $113.72(17)$ \\
\hline$C(3)-C(2)-C(1)$ & $102.47(16)$ \\
\hline$C(31)-C(3)-C(2)$ & $112.67(17)$ \\
\hline$C(31)-C(3)-C(4)$ & $113.54(17)$ \\
\hline$C(2)-C(3)-C(4)$ & $99.93(16)$ \\
\hline $\mathrm{N}-\mathrm{C}(4)-\mathrm{C}(41)$ & $122.07(18)$ \\
\hline $\mathrm{N}-\mathrm{C}(4)-\mathrm{C}(3)$ & $114.61(17)$ \\
\hline$C(41)-C(4)-C(3)$ & $123.31(18)$ \\
\hline$O(11)-C(11)-O(12)$ & $123.7(2)$ \\
\hline $\mathrm{O}(11)-\mathrm{C}(11)-\mathrm{C}(1)$ & $125.0(2)$ \\
\hline $\mathrm{O}(12)-\mathrm{C}(11)-\mathrm{C}(1)$ & $111.32(17)$ \\
\hline$O(21)-C(21)-O(22)$ & $124.4(2)$ \\
\hline $\mathrm{O}(21)-\mathrm{C}(21)-\mathrm{C}(2)$ & $126.0(2)$ \\
\hline$O(22)-C(21)-C(2)$ & $109.53(17)$ \\
\hline$O(22)-C(22)-C(23)$ & $108.9(2)$ \\
\hline$O(31)-C(31)-O(32)$ & $124.4(2)$ \\
\hline$O(31)-C(31)-C(3)$ & $123.91(18)$ \\
\hline$O(32)-C(31)-C(3)$ & $111.71(18)$ \\
\hline$O(32)-C(32)-C(33)$ & $110.8(2)$ \\
\hline$C(46)-C(41)-C(42)$ & $118.7(2)$ \\
\hline$C(46)-C(41)-C(4)$ & $121.04(19)$ \\
\hline
\end{tabular}




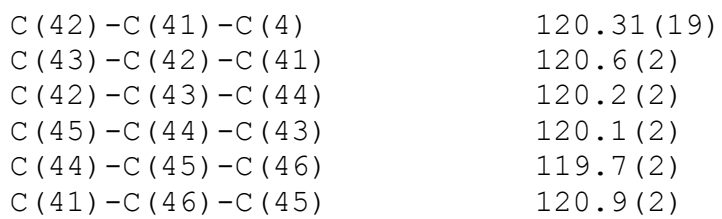

Symmetry transformations used to generate equivalent atoms:

Table 4. Anisotropic displacement parameters ( $A^{\wedge} 2 \times 10^{\wedge} 3$ ) for $2 / 4 a$. The anisotropic displacement factor exponent takes the form: $-2 \mathrm{pi}^{\wedge} 2\left[\mathrm{~h}^{\wedge} 2 \mathrm{a}^{\star \wedge} 2 \mathrm{U} 11+\ldots+2 \mathrm{~h} \mathrm{k} \mathrm{a}^{\star} \mathrm{b} \mathrm{b}^{\star} \mathrm{U} 12\right]$

\begin{tabular}{|c|c|c|c|c|c|c|}
\hline & U11 & U22 & U33 & U23 & U13 & U12 \\
\hline $\mathrm{N}$ & $33(1)$ & $28(1)$ & $30(1)$ & $9(1)$ & $13(1)$ & $9(1)$ \\
\hline$O(11)$ & $62(1)$ & $39(1)$ & $52(1)$ & $23(1)$ & $17(1)$ & $-2(1)$ \\
\hline$O(12)$ & $52(1)$ & $33(1)$ & $30(1)$ & $6(1)$ & $11(1)$ & $-8(1)$ \\
\hline$O(21)$ & $44(1)$ & $38(1)$ & $44(1)$ & $-2(1)$ & $20(1)$ & $8(1)$ \\
\hline$O(22)$ & $43(1)$ & $35(1)$ & $48(1)$ & $8(1)$ & $29(1)$ & $6(1)$ \\
\hline$O(31)$ & $41(1)$ & $38(1)$ & $38(1)$ & $15(1)$ & $6(1)$ & $4(1)$ \\
\hline$O(32)$ & $57(1)$ & $24(1)$ & $42(1)$ & $9(1)$ & $15(1)$ & $9(1)$ \\
\hline$C(1)$ & $35(1)$ & $26(1)$ & $29(1)$ & $8(1)$ & $14(1)$ & $8(1)$ \\
\hline$C(2)$ & $31(1)$ & $26(1)$ & $26(1)$ & $7(1)$ & $10(1)$ & $7(1)$ \\
\hline$C(3)$ & $31(1)$ & $25(1)$ & $26(1)$ & $6(1)$ & $10(1)$ & $6(1)$ \\
\hline C (4) & $28(1)$ & $26(1)$ & $25(1)$ & $5(1)$ & $5(1)$ & $6(1)$ \\
\hline$C(11)$ & $40(1)$ & $29(1)$ & $34(1)$ & $10(1)$ & $20(1)$ & $11(1)$ \\
\hline$C(12)$ & $56(2)$ & $42(2)$ & $39(1)$ & $2(1)$ & $12(1)$ & $-14(1)$ \\
\hline$C(13)$ & $46(1)$ & $37(1)$ & $36(1)$ & $15(1)$ & $15(1)$ & $14(1)$ \\
\hline$C(21)$ & $34(1)$ & $30(1)$ & $32(1)$ & 11 (1) & $13(1)$ & $10(1)$ \\
\hline C (22) & $56(2)$ & $58(2)$ & $58(2)$ & $14(1)$ & $40(1)$ & $14(1)$ \\
\hline C (23) & $73(2)$ & $79(2)$ & $88(2)$ & $44(2)$ & $50(2)$ & $28(2)$ \\
\hline$C(31)$ & $29(1)$ & $26(1)$ & $34(1)$ & $7(1)$ & $15(1)$ & $6(1)$ \\
\hline$C(32)$ & $69(2)$ & $30(1)$ & $66(2)$ & $22(1)$ & $21(2)$ & $18(1)$ \\
\hline$C(33)$ & $74(2)$ & $61(2)$ & $87(2)$ & $46(2)$ & $36(2)$ & $23(2)$ \\
\hline$C(41)$ & $27(1)$ & $32(1)$ & $31(1)$ & 11 (1) & $10(1)$ & $10(1)$ \\
\hline C ( 42) & $40(1)$ & $34(1)$ & $39(1)$ & $11(1)$ & $17(1)$ & $10(1)$ \\
\hline$C(43)$ & $47(1)$ & $51(2)$ & $48(2)$ & $15(1)$ & $27(1)$ & $19(1)$ \\
\hline$C(44)$ & $36(1)$ & $65(2)$ & $58(2)$ & $30(1)$ & $26(1)$ & $17(1)$ \\
\hline C (45) & $35(1)$ & $44(2)$ & $63(2)$ & $20(1)$ & $20(1)$ & $5(1)$ \\
\hline$C(46)$ & $32(1)$ & $37(1)$ & $49(1)$ & $10(1)$ & $16(1)$ & $8(1)$ \\
\hline
\end{tabular}

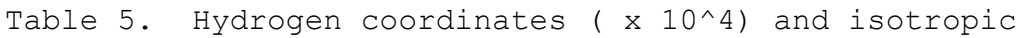
displacement parameters $\left(A^{\wedge} 2 \times 10^{\wedge} 3\right)$ for $\mathbf{2 / 4 a .}$

\begin{tabular}{lrrrr}
\hline & X & y & z & U (eq) \\
\hline H (2A) & -3535 & -2418 & 2213 & 34 \\
H (3A) & -178 & -565 & 3770 & 34 \\
H (12A) & -6465 & -6418 & -103 & 82 \\
H (12B) & -6806 & -6597 & 972 & 82 \\
H (12C) & -5561 & -7359 & 580 & 82
\end{tabular}




$\begin{array}{lrrrr}H(13 A) & -959 & -4492 & 4262 & 58 \\ H(13 B) & -2001 & -3473 & 4651 & 58 \\ H(13 C) & -200 & -2730 & 4623 & 58 \\ H(22 A) & -6021 & -1475 & 4445 & 65 \\ H(22 B) & -4554 & -1827 & 5319 & 65 \\ H(23 A) & -7219 & -3383 & 5020 & 106 \\ H(23 B) & -6144 & -4343 & 4604 & 106 \\ H(23 C) & -7603 & -4000 & 3725 & 106 \\ H(32 A) & -457 & 3477 & 2962 & 65 \\ H(32 B) & -1794 & 2220 & 1840 & 65 \\ H(33 A) & 483 & 3501 & 1505 & 102 \\ H(33 B) & 462 & 1822 & 1335 & 102 \\ H(33 C) & 1782 & 3101 & 2447 & 102 \\ H(42 A) & 561 & -4065 & 977 & 45 \\ H(43 A) & 2639 & -3504 & 236 & 55 \\ H(44 A) & 4437 & -1053 & 795 & 58 \\ H(45 A) & 4208 & 834 & 2135 & 57 \\ H(46 A) & 2110 & 277 & 2873 & 48\end{array}$

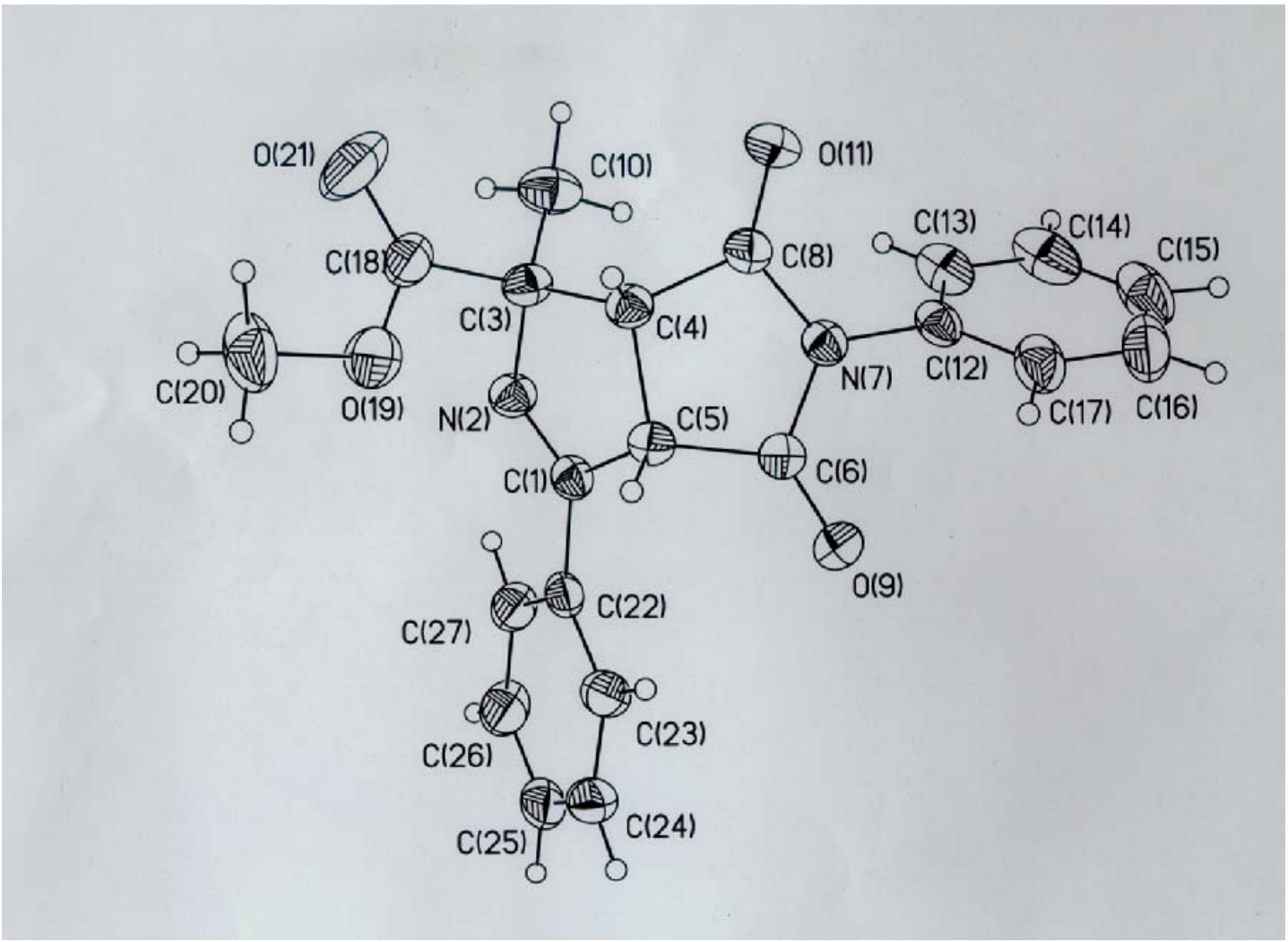

Table 1. Crystal data and structure refinement for

Compound 1 (crystallized from dichloromethane /octane). 
Identification code

Empirical formula

Formula weight

Temperature

Wavelength

Crystal system

Space group

Unit cell dimensions

Volume

Z

Density (calculated) tepe 013

C10.50 H9 N O2

181.19

$173(2) \mathrm{K}$

$0.71073 \mathrm{~A}$

Ortho.

P2 (1) 2 (1) 2(1)

$$
\begin{aligned}
& \mathrm{a}=6.7048(13) \mathrm{A} \\
& \mathrm{b}=13.522(3) \mathrm{A} \\
& \mathrm{c}=19.685(4) \mathrm{A} \\
& \text { alpha }=90 \mathrm{deg} . \\
& \text { beta }=90 \mathrm{deg} . \\
& \text { gamma }=90 \mathrm{deg} .
\end{aligned}
$$

$1784.7(6) A^{\wedge} 3$

8

$1.349 \mathrm{Mg} / \mathrm{m}^{\wedge} 3$ 


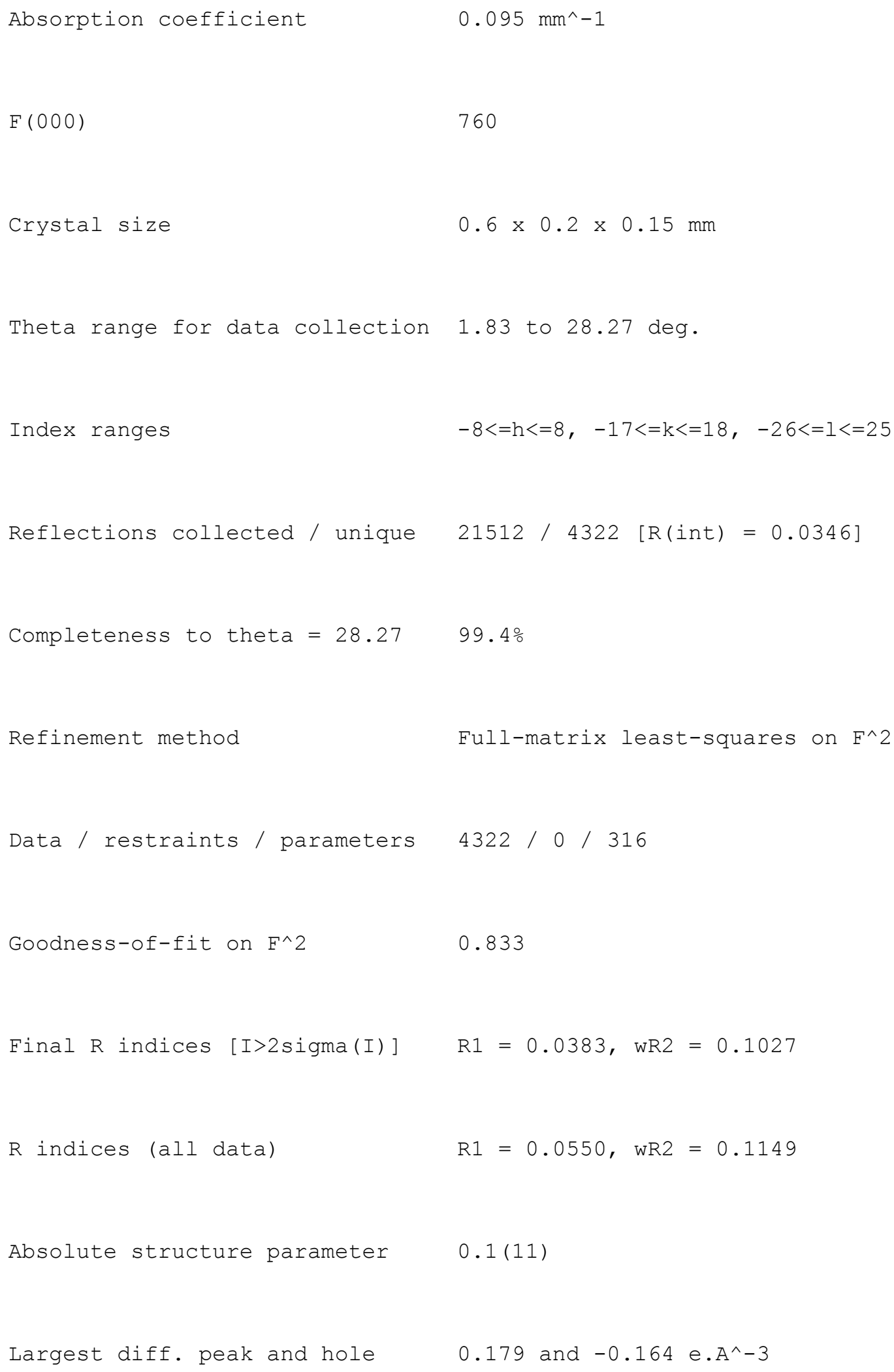


Table 2. Atomic coordinates ( $x$ 10^4), equivalent isotropic displacement parameters $\left(A^{\wedge} 2 \times 10^{\wedge} 3\right)$, and occupancies for compound 1 .

\begin{tabular}{|c|c|c|c|c|c|}
\hline & $x$ & y & $\mathrm{z}$ & $\mathrm{U}(\mathrm{eq})$ & Occ. \\
\hline$C(1)$ & $6754(2)$ & $5346(1)$ & $-946(1)$ & $26(1)$ & 1 \\
\hline $\mathrm{N}(2)$ & $5259(2)$ & $5203(1)$ & $-1335(1)$ & $30(1)$ & 1 \\
\hline$C(3)$ & $4728(3)$ & $4146(1)$ & $-1357(1)$ & $30(1)$ & 1 \\
\hline$C(4)$ & $6045(2)$ & $3625(1)$ & $-806(1)$ & $27(1)$ & 1 \\
\hline$C(5)$ & $7519(3)$ & $4423(1)$ & $-578(1)$ & $26(1)$ & 1 \\
\hline$C(6)$ & $7252(2)$ & $4505(1)$ & $188(1)$ & $26(1)$ & 1 \\
\hline$N(7)$ & $5590(2)$ & $3937(1)$ & $367(1)$ & $28(1)$ & 1 \\
\hline$C(8)$ & $4941(3)$ & $3331(1)$ & $-164(1)$ & $31(1)$ & 1 \\
\hline$O(9)$ & $8237(2)$ & $4977(1)$ & $580(1)$ & $36(1)$ & 1 \\
\hline$C(10)$ & $2481(3)$ & $4044(2)$ & $-1266(1)$ & $43(1)$ & 1 \\
\hline$O(11)$ & $3719(2)$ & $2685(1)$ & $-104(1)$ & $46(1)$ & 1 \\
\hline C (12) & $4829(3)$ & $3883(1)$ & $1049(1)$ & $31(1)$ & 1 \\
\hline$C(13)$ & $2850(3)$ & $4126(1)$ & $1169(1)$ & $41(1)$ & 1 \\
\hline C (14) & $2130(4)$ & $4109(2)$ & $1829(1)$ & $55(1)$ & 1 \\
\hline$C(15)$ & $3380(5)$ & $3847(2)$ & $2360(1)$ & $59(1)$ & 1 \\
\hline$C(16)$ & $5341(5)$ & $3602(2)$ & $2238(1)$ & $56(1)$ & 1 \\
\hline C (17) & $6076(4)$ & $3614(1)$ & $1577(1)$ & $42(1)$ & 1 \\
\hline$C(18)$ & $5369(3)$ & $3766(1)$ & $-2057(1)$ & $36(1)$ & 1 \\
\hline$O(19)$ & $7234(2)$ & $4018(1)$ & $-2185(1)$ & $53(1)$ & 1 \\
\hline$C(20)$ & $8056(5)$ & $3740(2)$ & $-2838(1)$ & $60(1)$ & 1 \\
\hline$O(21)$ & $4349(3)$ & $3289(2)$ & $-2424(1)$ & $75(1)$ & 1 \\
\hline
\end{tabular}




$\begin{array}{lrllll}C(22) & 7681(3) & 6336(1) & -877(1) & 29(1) & 1 \\ C(23) & 9575(3) & 6458(1) & -596(1) & 34(1) & 1 \\ C(24) & 10431(4) & 7394(2) & -567(1) & 43(1) & 1 \\ C(25) & 9379(4) & 8209(2) & -796(1) & 46(1) & 1 \\ \text { C (26) } & 7493(4) & 8093(2) & -1074(1) & 49(1) & 1 \\ C(27) & 6654(3) & 7164(1) & -1121(1) & 38(1) & 1\end{array}$

$\mathrm{U}($ eq) is defined as one third of the trace of the orthogonalized Uij tensor.

Table 3. Bond lengths $[\mathrm{A}]$ and angles [deg] for Compound 1 .

$\begin{array}{ll}C(1)-N(2) & 1.276(2) \\ C(1)-C(22) & 1.483(2) \\ C(1)-C(5) & 1.530(2) \\ N(2)-C(3) & 1.473(2) \\ C(3)-C(10) & 1.524(3) \\ C(3)-C(18) & 1.531(2) \\ C(3)-C(4) & 1.568(2) \\ C(4)-C(8) & 1.517(2) \\ C(4)-C(5) & 1.530(2) \\ C(5)-C(6) & 1.523(2) \\ C(6)-O(9) & 1.200(2) \\ C(6)-N(7) & 1.398(2) \\ N(7)-C(8) & 1.398(2)\end{array}$




\begin{tabular}{|c|c|}
\hline $\mathrm{N}(7)-\mathrm{C}(12)$ & $1.439(2)$ \\
\hline$C(8)-O(11)$ & $1.203(2)$ \\
\hline$C(12)-C(13)$ & $1.387(3)$ \\
\hline$C(12)-C(17)$ & $1.383(3)$ \\
\hline$C(13)-C(14)$ & $1.385(3)$ \\
\hline$C(14)-C(15)$ & $1.387(4)$ \\
\hline$C(15)-C(16)$ & $1.378(4)$ \\
\hline$C(16)-C(17)$ & $1.391(3)$ \\
\hline$C(18)-O(21)$ & $1.187(2)$ \\
\hline $\mathrm{C}(18)-\mathrm{O}(19)$ & $1.320(3)$ \\
\hline$O(19)-C(20)$ & $1.449(3)$ \\
\hline$C(22)-C(27)$ & $1.398(3)$ \\
\hline$C(22)-C(23)$ & $1.395(3)$ \\
\hline$C(23)-C(24)$ & $1.391(3)$ \\
\hline$C(24)-C(25)$ & $1.384(3)$ \\
\hline$C(25)-C(26)$ & $1.388(4)$ \\
\hline$C(26)-C(27)$ & $1.380(3)$ \\
\hline $\mathrm{N}(2)-\mathrm{C}(1)-\mathrm{C}(22)$ & $121.36(15)$ \\
\hline $\mathrm{N}(2)-\mathrm{C}(1)-\mathrm{C}(5)$ & $115.06(14)$ \\
\hline$C(22)-C(1)-C(5)$ & $123.57(14)$ \\
\hline $\mathrm{C}(1)-\mathrm{N}(2)-\mathrm{C}(3)$ & $110.77(13)$ \\
\hline$N(2)-C(3)-C(10)$ & $108.84(15)$ \\
\hline $\mathrm{N}(2)-\mathrm{C}(3)-\mathrm{C}(18)$ & $106.55(14)$ \\
\hline$C(10)-C(3)-C(18)$ & $110.72(16)$ \\
\hline$N(2)-C(3)-C(4)$ & $106.19(13)$ \\
\hline$C(10)-C(3)-C(4)$ & $115.76(16)$ \\
\hline$C(18)-C(3)-C(4)$ & $108.31(14)$ \\
\hline$C(8)-C(4)-C(5)$ & $104.88(13)$ \\
\hline
\end{tabular}




$$
\begin{aligned}
& \mathrm{C}(8)-\mathrm{C}(4)-\mathrm{C}(3) \quad 114.80(14) \\
& C(5)-C(4)-C(3) \quad 104.43(13) \\
& C(1)-C(5)-C(6) \quad 111.68(13) \\
& C(1)-C(5)-C(4) \quad 102.74(13) \\
& C(6)-C(5)-C(4) \quad 105.37(13) \\
& \mathrm{O}(9)-\mathrm{C}(6)-\mathrm{N}(7) \quad 124.72(15) \\
& O(9)-C(6)-C(5) \quad 127.63(16) \\
& N(7)-C(6)-C(5) \quad 107.63(13) \\
& \mathrm{C}(6)-\mathrm{N}(7)-\mathrm{C}(8) \quad 112.46(13) \\
& \mathrm{C}(6)-\mathrm{N}(7)-\mathrm{C}(12) \quad 123.06(13) \\
& \mathrm{C}(8)-\mathrm{N}(7)-\mathrm{C}(12) \quad 123.89(14) \\
& \mathrm{O}(11)-\mathrm{C}(8)-\mathrm{N}(7) \quad 124.35(16) \\
& \mathrm{O}(11)-\mathrm{C}(8)-\mathrm{C}(4) \quad 127.20(16) \\
& \mathrm{N}(7)-\mathrm{C}(8)-\mathrm{C}(4) \quad 108.45(13) \\
& C(13)-C(12)-C(17) \quad 120.87(18) \\
& \mathrm{C}(13)-\mathrm{C}(12)-\mathrm{N}(7) \quad 119.10(17) \\
& \mathrm{C}(17)-\mathrm{C}(12)-\mathrm{N}(7) \quad 120.01(16) \\
& C(14)-C(13)-C(12) \quad 119.3(2) \\
& C(13)-C(14)-C(15) \quad 120.1(2) \\
& C(16)-C(15)-C(14) \quad 120.4(2) \\
& C(15)-C(16)-C(17) \quad 120.0(2) \\
& \mathrm{C}(12)-\mathrm{C}(17)-\mathrm{C}(16) \quad 119.4(2) \\
& O(21)-C(18)-O(19) \quad 124.69(19) \\
& O(21)-C(18)-C(3) \quad 124.69(19) \\
& O(19)-C(18)-C(3) \quad 110.59(15) \\
& \mathrm{C}(18)-\mathrm{O}(19)-\mathrm{C}(20) \quad 117.59(19) \\
& C(27)-C(22)-C(23) \quad 119.32(17) \\
& C(27)-C(22)-C(1) \quad 119.02(16)
\end{aligned}
$$




$$
\begin{array}{ll}
C(23)-C(22)-C(1) & 121.63(16) \\
C(24)-C(23)-C(22) & 119.99(19) \\
C(25)-C(24)-C(23) & 120.1(2) \\
C(26)-C(25)-C(24) & 120.19(18) \\
C(25)-C(26)-C(27) & 120.0(2) \\
C(26)-C(27)-C(22) & 120.4(2)
\end{array}
$$

\begin{tabular}{|c|c|c|c|c|c|c|}
\hline C (1) & $28(1)$ & $25(1)$ & $26(1)$ & $0(1)$ & $3(1)$ & $0(1)$ \\
\hline$N(2)$ & $34(1)$ & $24(1)$ & $33(1)$ & $1(1)$ & $-3(1)$ & $-1(1)$ \\
\hline$C(3)$ & $30(1)$ & $24(1)$ & $34(1)$ & $-1(1)$ & $-4(1)$ & $-2(1)$ \\
\hline$C(4)$ & $29(1)$ & $22(1)$ & $29(1)$ & $-3(1)$ & $1(1)$ & $-1(1)$ \\
\hline$C(5)$ & $23(1)$ & $25(1)$ & $29(1)$ & $-1(1)$ & $2(1)$ & $1(1)$ \\
\hline$C(6)$ & $24(1)$ & $22(1)$ & $31(1)$ & $2(1)$ & $-2(1)$ & $4(1)$ \\
\hline $\mathrm{N}(7)$ & $29(1)$ & $25(1)$ & $28(1)$ & $0(1)$ & $3(1)$ & $0(1)$ \\
\hline$C(8)$ & $35(1)$ & $26(1)$ & $31(1)$ & $0(1)$ & $0(1)$ & $-4(1)$ \\
\hline$O(9)$ & $39(1)$ & $38(1)$ & $33(1)$ & $-1(1)$ & $-6(1)$ & $-7(1)$ \\
\hline$C(10)$ & $30(1)$ & 37 (1) & $61(1)$ & $3(1)$ & $-6(1)$ & $-3(1)$ \\
\hline $\mathrm{O}(11)$ & $53(1)$ & $42(1)$ & $42(1)$ & $1(1)$ & $4(1)$ & $-23(1)$ \\
\hline
\end{tabular}

Symmetry transformations used to generate equivalent atoms:

Table 4. Anisotropic displacement parameters ( $\left.A^{\wedge} 2 \times 10^{\wedge} 3\right)$ for tepe 013

$\begin{array}{lllll}\text { U11 } & \text { U22 } & \text { U33 } & \text { U23 } & \text { U13 }\end{array}$




\begin{tabular}{|c|c|c|c|c|c|c|}
\hline C (12) & $40(1)$ & 21 (1) & $31(1)$ & $0(1)$ & $7(1)$ & $-2(1)$ \\
\hline$C(13)$ & $43(1)$ & 32 (1) & $49(1)$ & $-1(1)$ & $14(1)$ & $0(1)$ \\
\hline C (14) & $63(1)$ & $37(1)$ & $65(2)$ & $-7(1)$ & $32(1)$ & $-4(1)$ \\
\hline C (15) & $100(2)$ & $37(1)$ & $40(1)$ & $-4(1)$ & $28(1)$ & $-11(1)$ \\
\hline$C(16)$ & $90(2)$ & $44(1)$ & $32(1)$ & $5(1)$ & $-3(1)$ & $-4(1)$ \\
\hline C (17) & $58(1)$ & 31 (1) & $37(1)$ & $4(1)$ & $1(1)$ & $1(1)$ \\
\hline C (18) & $48(1)$ & $30(1)$ & $30(1)$ & $-1(1)$ & $-7(1)$ & $-1(1)$ \\
\hline O (19) & $60(1)$ & $60(1)$ & $40(1)$ & $-18(1)$ & $16(1)$ & $-14(1)$ \\
\hline$C(20)$ & $91(2)$ & $50(1)$ & $38(1)$ & $-4(1)$ & $24(1)$ & $2(1)$ \\
\hline$O(21)$ & $80(1)$ & $96(1)$ & $51(1)$ & $-31(1)$ & $-12(1)$ & $-23(1)$ \\
\hline C (22) & $38(1)$ & $26(1)$ & $22(1)$ & $0(1)$ & $4(1)$ & $-4(1)$ \\
\hline C (23) & $35(1)$ & $33(1)$ & $35(1)$ & $-2(1)$ & $4(1)$ & $-5(1)$ \\
\hline C (24) & $46(1)$ & $47(1)$ & $36(1)$ & $-8(1)$ & $5(1)$ & $-18(1)$ \\
\hline$C(25)$ & $74(2)$ & 31 (1) & $32(1)$ & $1(1)$ & $2(1)$ & $-21(1)$ \\
\hline$C(26)$ & $79(2)$ & $29(1)$ & $40(1)$ & $7(1)$ & $-14(1)$ & $-10(1)$ \\
\hline$C(27)$ & $54(1)$ & $28(1)$ & $33(1)$ & $4(1)$ & $-9(1)$ & $-5(1)$ \\
\hline
\end{tabular}

The anisotropic displacement factor exponent takes the form: $-2 \mathrm{pi}^{\wedge} 2\left[\mathrm{~h}^{\wedge} 2 \mathrm{a}^{\star \wedge} 2 \mathrm{U} 11+\ldots+2 \mathrm{~h} k \mathrm{a}^{\star} \mathrm{b} \mathrm{b}^{\star} \mathrm{U} 12\right]$

Table 5. Hydrogen coordinates ( $\mathrm{x}$ 10^4), isotropic displacement parameters $\left(A^{\wedge} 2 \times 10^{\wedge} 3\right)$, and occupancies for tepe 013

$\mathrm{x}$ Y

z

$\mathrm{U}(\mathrm{eq})$

Occ. 


\begin{tabular}{|c|c|c|c|c|c|}
\hline $\mathrm{H}(4)$ & $6730(30)$ & $3033(13)$ & $-967(9)$ & $19(4)$ & 1 \\
\hline $\mathrm{H}(5)$ & $8870(40)$ & $4316(17)$ & $-695(11)$ & $40(6)$ & 1 \\
\hline $\mathrm{H}(10 \mathrm{~A})$ & $2130(40)$ & $4317(18)$ & $-804(12)$ & $45(6)$ & 1 \\
\hline $\mathrm{H}(10 \mathrm{~B})$ & $1810(40)$ & $4408(18)$ & $-1631(13)$ & $53(7)$ & 1 \\
\hline $\mathrm{H}(10 \mathrm{C})$ & $2080(40)$ & $3330(20)$ & $-1277(14)$ & $66(8)$ & 1 \\
\hline $\mathrm{H}(13)$ & $1970(30)$ & $4303(16)$ & $820(12)$ & $39(6)$ & 1 \\
\hline $\mathrm{H}(14)$ & $670(50)$ & $4290(30)$ & $1933(17)$ & $95(10)$ & 1 \\
\hline H (15) & $2880(40)$ & $3860(20)$ & $2848(15)$ & $69(8)$ & 1 \\
\hline $\mathrm{H}(16)$ & $6140(40)$ & $3400(20)$ & $2581(17)$ & $77(9)$ & 1 \\
\hline $\mathrm{H}(17)$ & $7550(40)$ & $3498(17)$ & $1448(12)$ & $45(6)$ & 1 \\
\hline $\mathrm{H}(20 \mathrm{~A})$ & $7740(40)$ & $3040(20)$ & $-2918(13)$ & $65(8)$ & 1 \\
\hline $\mathrm{H}(20 \mathrm{~B})$ & $7420(50)$ & $4150(30)$ & $-3183(18)$ & $99(11)$ & 1 \\
\hline $\mathrm{H}(20 \mathrm{C})$ & $9490(60)$ & $3890(30)$ & $-2824(19)$ & $103(12)$ & 1 \\
\hline $\mathrm{H}(23)$ & $10280(30)$ & $5907(17)$ & $-423(11)$ & $40(6)$ & 1 \\
\hline $\mathrm{H}(24)$ & $11730(40)$ & $7458(17)$ & $-403(11)$ & $44(6)$ & 1 \\
\hline $\mathrm{H}(25)$ & $9930(30)$ & $8878(18)$ & $-763(11)$ & $47(6)$ & 1 \\
\hline $\mathrm{H}(26)$ & $6790(40)$ & $8650(20)$ & $-1236(14)$ & $64(7)$ & 1 \\
\hline $\mathrm{H}(27)$ & $5390(40)$ & $7024(17)$ & $-1295(11)$ & $43(6)$ & 1 \\
\hline
\end{tabular}




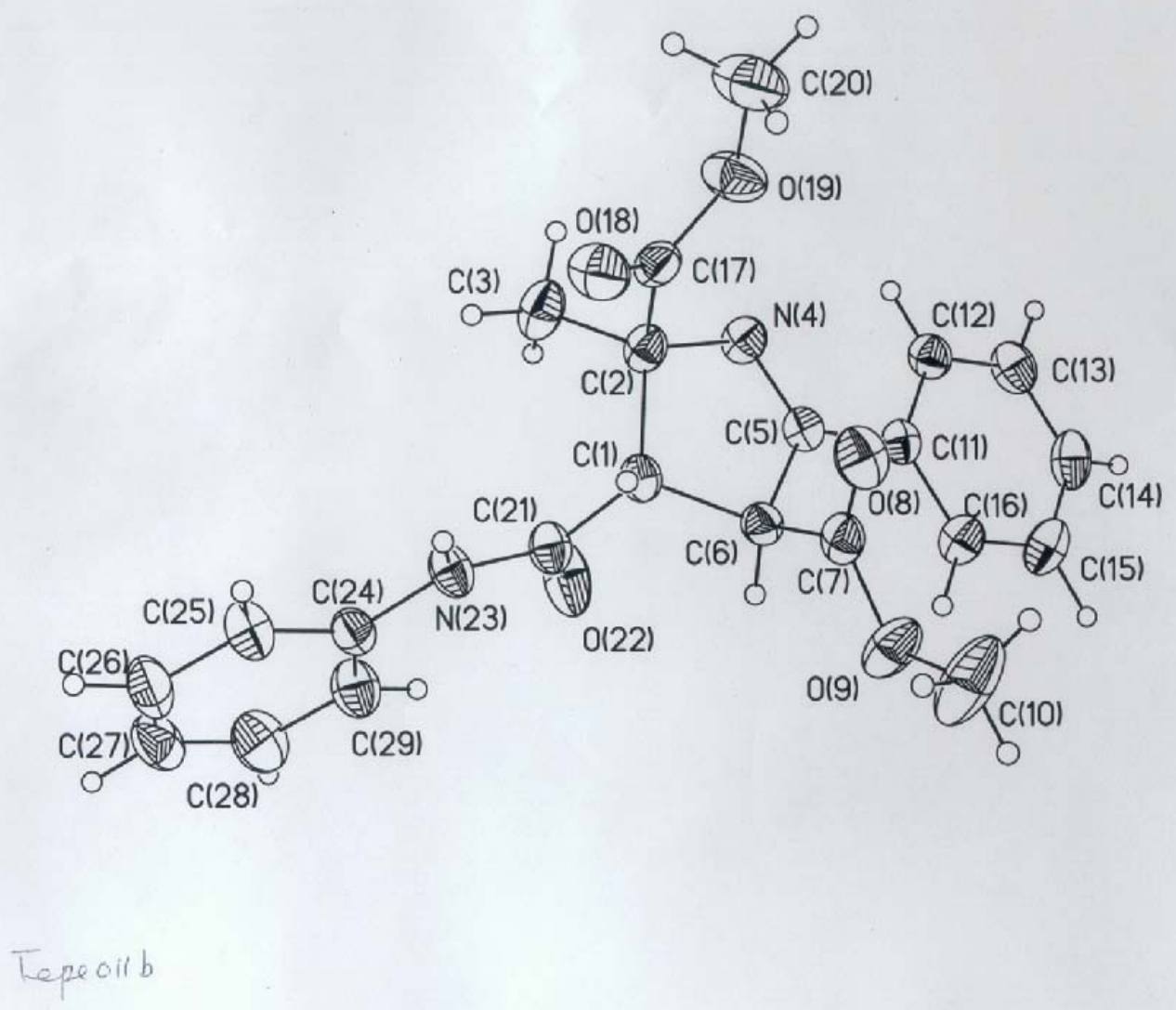

Table 1. Crystal data and structure refinement for Compound 12 (crystallized from dichloromethane /octane). 
Formula weight

Temperature

Wavelength

Crystal system

space group

Unit cell dimensions

Volume

Z

Density (calculated)

Absorption coefficient

F $(000)$

Crystal size
394.42

$173(2) \mathrm{K}$

$0.71073 \mathrm{~A}$

Triclinic

$\mathrm{P}-1$

$\mathrm{a}=8.9567(18) \mathrm{A}$
$\mathrm{b}=10.444(2) \mathrm{A}$
$\mathrm{c}=11.475(2) \mathrm{A}$
alpha $=99.06(3) \mathrm{deg}$.
beta $=104.61(3) \mathrm{deg}$.
gamma $=99.54(3) \mathrm{deg}$.

$1001.7(3) A^{\wedge} 3$

2

$1.308 \mathrm{Mg} / \mathrm{m}^{\wedge} 3$

$0.094 \mathrm{~mm}^{\wedge}-1$

416

$0.4 \times 0.3 \times 0.25 \mathrm{~mm}$ 


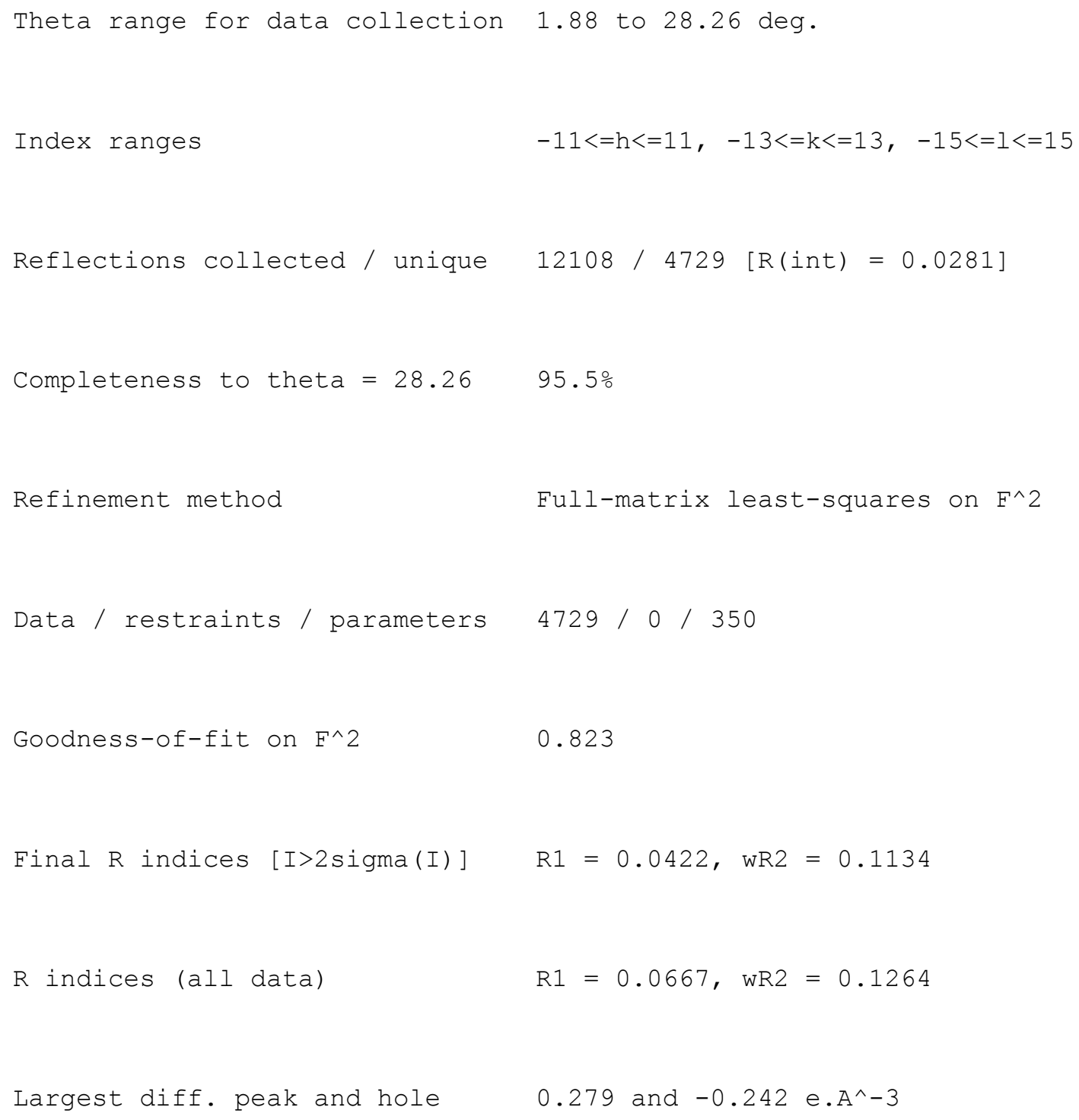


Table 2. Atomic coordinates ( $\mathrm{x}$ 10^4), equivalent isotropic displacement parameters $\left(A^{\wedge} 2 \times 10^{\wedge} 3\right)$, and occupancies for Compound 12 .

\begin{tabular}{|c|c|c|c|c|c|}
\hline & $x$ & Y & $\mathrm{z}$ & $\mathrm{U}(\mathrm{eq})$ & Occ. \\
\hline$C(1)$ & $47(2)$ & $5728(1)$ & $1938(1)$ & $27(1)$ & 1 \\
\hline$C(2)$ & $1830(2)$ & $5917(1)$ & $2686(1)$ & $29(1)$ & 1 \\
\hline$C(3)$ & $2156(2)$ & $4947(2)$ & $3556(2)$ & $42(1)$ & 1 \\
\hline$N(4)$ & $2321(1)$ & $7279(1)$ & $3419(1)$ & $30(1)$ & 1 \\
\hline$C(5)$ & $1257(2)$ & $7935(1)$ & $3103(1)$ & $25(1)$ & 1 \\
\hline$C(6)$ & $-210(2)$ & $7167(1)$ & $2065(1)$ & $26(1)$ & 1 \\
\hline$C(7)$ & $-254(2)$ & $7637(1)$ & $880(1)$ & $29(1)$ & 1 \\
\hline$O(8)$ & $764(1)$ & $7569(1)$ & $363(1)$ & $39(1)$ & 1 \\
\hline$O(9)$ & $-1525(1)$ & $8116(1)$ & $474(1)$ & $44(1)$ & 1 \\
\hline$C(10)$ & $-1751(4)$ & $8467(2)$ & $-728(2)$ & $68(1)$ & 1 \\
\hline $\mathrm{C}(11)$ & $1433(2)$ & $9336(1)$ & $3696(1)$ & $26(1)$ & 1 \\
\hline C (12) & $2824(2)$ & $9971(1)$ & $4620(1)$ & $31(1)$ & 1 \\
\hline C (13) & $3019(2)$ & $11280(2)$ & $5218(1)$ & $37(1)$ & 1 \\
\hline$C(14)$ & $1834(2)$ & $11959(2)$ & $4902(1)$ & $38(1)$ & 1 \\
\hline$C(15)$ & $439(2)$ & $11338(2)$ & $3995(1)$ & $40(1)$ & 1 \\
\hline$C(16)$ & $240(2)$ & $10029(1)$ & $3388(1)$ & $33(1)$ & 1 \\
\hline C (17) & $2807(2)$ & $5709(1)$ & $1778(1)$ & $30(1)$ & 1 \\
\hline$O(18)$ & $2411(1)$ & $4823(1)$ & $883(1)$ & $42(1)$ & 1 \\
\hline O (19) & $4170(1)$ & $6595(1)$ & $2106(1)$ & $45(1)$ & 1 \\
\hline C (20) & $5175(2)$ & $6428(2)$ & $1303(2)$ & $59(1)$ & 1 \\
\hline
\end{tabular}




$\begin{array}{llllll}\text { C (21) } & -1101(2) & 4905(1) & 2454(1) & 31(1) & 1 \\ \text { O(22) } & -1446(2) & 5370(1) & 3364(1) & 53(1) & 1 \\ \text { N (23) } & -1690(1) & 3637(1) & 1806(1) & 31(1) & 1 \\ \text { C (24) } & -2764(2) & 2626(1) & 2058(1) & 31(1) & 1 \\ \text { C (25) } & -3008(2) & 1348(2) & 1372(2) & 40(1) & 1 \\ \text { C (26) } & -4030(2) & 310(2) & 1572(2) & 45(1) & 1 \\ \text { C (27) } & -4818(2) & 523(2) & 2440(2) & 46(1) & 1 \\ \text { C (28) } & -4589(2) & 1797(2) & 3111(2) & 50(1) & 1 \\ \text { C (29) } & -3565(2) & 2855(2) & 2934(2) & 42(1) & 1\end{array}$

$\mathrm{U}(e q)$ is defined as one third of the trace of the orthogonalized Uij tensor.

Table 3. Bond lengths [A] and angles [deg] for Compound 12 .

$\begin{array}{ll}C(1)-C(21) & 1.523(2) \\ C(1)-C(6) & 1.5476(19) \\ C(1)-C(2) & 1.5738(19) \\ C(2)-N(4) & 1.4675(17) \\ C(2)-C(17) & 1.533(2) \\ C(2)-C(3) & 1.542(2) \\ N(4)-C(5) & 1.2763(18) \\ C(5)-C(11) & 1.4782(18) \\ C(5)-C(6) & 1.5320(19) \\ C(6)-C(7) & 1.510(2) \\ C(7)-O(8) & 1.2118(17) \\ C(7)-O(9) & 1.3261(18)\end{array}$




\begin{tabular}{|c|c|}
\hline $\mathrm{O}(9)-\mathrm{C}(10)$ & $1.456(2)$ \\
\hline$C(11)-C(16)$ & $1.392(2)$ \\
\hline$C(11)-C(12)$ & $1.398(2)$ \\
\hline$C(12)-C(13)$ & $1.391(2)$ \\
\hline$C(13)-C(14)$ & $1.378(2)$ \\
\hline$C(14)-C(15)$ & $1.388(2)$ \\
\hline$C(15)-C(16)$ & $1.394(2)$ \\
\hline $\mathrm{C}(17)-\mathrm{O}(18)$ & $1.2022(17)$ \\
\hline $\mathrm{C}(17)-\mathrm{O}(19)$ & $1.3308(18)$ \\
\hline$O(19)-C(20)$ & $1.452(2)$ \\
\hline$C(21)-O(22)$ & $1.2148(17)$ \\
\hline $\mathrm{C}(21)-\mathrm{N}(23)$ & $1.3580(18)$ \\
\hline $\mathrm{N}(23)-\mathrm{C}(24)$ & $1.4222(18)$ \\
\hline$C(24)-C(29)$ & $1.388(2)$ \\
\hline$C(24)-C(25)$ & $1.391(2)$ \\
\hline$C(25)-C(26)$ & $1.386(2)$ \\
\hline$C(26)-C(27)$ & $1.370(3)$ \\
\hline$C(27)-C(28)$ & $1.383(3)$ \\
\hline$C(28)-C(29)$ & $1.390(2)$ \\
\hline$C(21)-C(1)-C(6)$ & $109.67(11)$ \\
\hline$C(21)-C(1)-C(2)$ & $113.64(11)$ \\
\hline$C(6)-C(1)-C(2)$ & $103.11(11)$ \\
\hline $\mathrm{N}(4)-\mathrm{C}(2)-\mathrm{C}(17)$ & $110.80(11)$ \\
\hline$N(4)-C(2)-C(3)$ & $108.88(11)$ \\
\hline$C(17)-C(2)-C(3)$ & $106.75(12)$ \\
\hline$N(4)-C(2)-C(1)$ & $106.65(11)$ \\
\hline$C(17)-C(2)-C(1)$ & $108.89(11)$ \\
\hline$C(3)-C(2)-C(1)$ & $114.91(13)$ \\
\hline
\end{tabular}




$$
\begin{aligned}
& \mathrm{C}(5)-\mathrm{N}(4)-\mathrm{C}(2) \quad 110.55(11) \\
& \mathrm{N}(4)-\mathrm{C}(5)-\mathrm{C}(11) \quad 122.18(12) \\
& N(4)-C(5)-C(6) \quad 115.24(11) \\
& C(11)-C(5)-C(6) \quad 122.58(12) \\
& C(7)-C(6)-C(5) \quad 111.20(11) \\
& \mathrm{C}(7)-\mathrm{C}(6)-\mathrm{C}(1) \quad 110.29(11) \\
& C(5)-C(6)-C(1) \quad 102.18(11) \\
& O(8)-C(7)-O(9) \quad 124.39(13) \\
& O(8)-C(7)-C(6) \quad 123.05(13) \\
& O(9)-C(7)-C(6) \quad 112.55(12) \\
& C(7)-O(9)-C(10) \quad 116.38(16) \\
& C(16)-C(11)-C(12) \quad 119.06(13) \\
& C(16)-C(11)-C(5) \quad 122.00(12) \\
& C(12)-C(11)-C(5) \quad 118.91(12) \\
& C(13)-C(12)-C(11) \quad 120.56(14) \\
& \text { C (14) -C (13)-C (12) } 119.92(14) \\
& C(13)-C(14)-C(15) \quad 120.22(14) \\
& C(14)-C(15)-C(16) \quad 120.16(15) \\
& C(11)-C(16)-C(15) \quad 120.08(14) \\
& O(18)-C(17)-O(19) \quad 123.34(14) \\
& O(18)-C(17)-C(2) \quad 123.93(13) \\
& \mathrm{O}(19)-\mathrm{C}(17)-\mathrm{C}(2) \quad 112.70(12) \\
& C(17)-O(19)-C(20) \quad 115.47(13) \\
& \mathrm{O}(22)-\mathrm{C}(21)-\mathrm{N}(23) \quad 124.14(13) \\
& O(22)-C(21)-C(1) \quad 121.61(13) \\
& \mathrm{N}(23)-\mathrm{C}(21)-\mathrm{C}(1) \quad 114.24(12) \\
& \mathrm{C}(21)-\mathrm{N}(23)-\mathrm{C}(24) \quad 127.77(12) \\
& C(29)-C(24)-C(25) \quad 119.47(14)
\end{aligned}
$$




$$
\begin{array}{ll}
C(29)-C(24)-N(23) & 123.59(13) \\
C(25)-C(24)-N(23) & 116.94(13) \\
C(26)-C(25)-C(24) & 120.17(15) \\
C(27)-C(26)-C(25) & 120.84(16) \\
C(26)-C(27)-C(28) & 118.90(15) \\
C(27)-C(28)-C(29) & 121.48(17) \\
C(24)-C(29)-C(28) & 119.13(16)
\end{array}
$$

\begin{tabular}{|c|c|c|c|c|c|c|}
\hline & $\mathrm{U} 11$ & $\mathrm{U} 22$ & U33 & U23 & U13 & $\mathrm{U} 12$ \\
\hline$C(1)$ & $30(1)$ & $23(1)$ & $25(1)$ & $1(1)$ & $7(1)$ & $3(1)$ \\
\hline C (2) & $33(1)$ & $24(1)$ & $26(1)$ & $1(1)$ & $3(1)$ & $7(1)$ \\
\hline$C(3)$ & $55(1)$ & $33(1)$ & $36(1)$ & $10(1)$ & $5(1)$ & $14(1)$ \\
\hline$N(4)$ & $33(1)$ & $26(1)$ & $25(1)$ & $-1(1)$ & $3(1)$ & $7(1)$ \\
\hline$C(5)$ & $28(1)$ & $25(1)$ & $21(1)$ & $3(1)$ & $7(1)$ & $3(1)$ \\
\hline$C(6)$ & $25(1)$ & $24(1)$ & $28(1)$ & $3(1)$ & $6(1)$ & $3(1)$ \\
\hline$C(7)$ & $34(1)$ & $22(1)$ & $26(1)$ & $0(1)$ & $2(1)$ & $3(1)$ \\
\hline$O(8)$ & $47(1)$ & $40(1)$ & $31(1)$ & $8(1)$ & $14(1)$ & $3(1)$ \\
\hline$O(9)$ & $54(1)$ & $40(1)$ & $31(1)$ & $3(1)$ & $-3(1)$ & $22(1)$ \\
\hline$C(10)$ & $109(2)$ & $54(1)$ & $32(1)$ & $9(1)$ & $-7(1)$ & $40(1)$ \\
\hline$C(11)$ & $31(1)$ & $24(1)$ & $25(1)$ & $4(1)$ & $11(1)$ & $4(1)$ \\
\hline$C(12)$ & $32(1)$ & $28(1)$ & $32(1)$ & $2(1)$ & $9(1)$ & $4(1)$ \\
\hline
\end{tabular}

Symmetry transformations used to generate equivalent atoms:

Table 4. Anisotropic displacement parameters ( $A^{\wedge} 2 \times 10^{\wedge} 3$ ) for Compound 12 . 


\begin{tabular}{|c|c|c|c|c|c|c|}
\hline$C(13)$ & $42(1)$ & $29(1)$ & $34(1)$ & $-2(1)$ & $10(1)$ & $-1(1)$ \\
\hline$C(14)$ & $59(1)$ & $24(1)$ & $32(1)$ & $2(1)$ & $20(1)$ & $7(1)$ \\
\hline C (15) & $55(1)$ & $32(1)$ & $37(1)$ & $9(1)$ & $14(1)$ & $20(1)$ \\
\hline$C(16)$ & $39(1)$ & $31(1)$ & $29(1)$ & $4(1)$ & $7(1)$ & $10(1)$ \\
\hline C (17) & $28(1)$ & $25(1)$ & $33(1)$ & $1(1)$ & $1(1)$ & $9(1)$ \\
\hline$O(18)$ & $38(1)$ & $38(1)$ & $41(1)$ & $-10(1)$ & $11(1)$ & $4(1)$ \\
\hline O (19) & $30(1)$ & $45(1)$ & $50(1)$ & $-12(1)$ & $10(1)$ & $-1(1)$ \\
\hline$C(20)$ & $37(1)$ & $61(1)$ & $72(1)$ & $-6(1)$ & $22(1)$ & $2(1)$ \\
\hline C (21) & $38(1)$ & $25(1)$ & $30(1)$ & $3(1)$ & $12(1)$ & $4(1)$ \\
\hline$O(22)$ & $82(1)$ & $31(1)$ & $51(1)$ & $-2(1)$ & $42(1)$ & $0(1)$ \\
\hline $\mathrm{N}(23)$ & $36(1)$ & $27(1)$ & $29(1)$ & $1(1)$ & $14(1)$ & $-1(1)$ \\
\hline C (24) & $30(1)$ & $30(1)$ & $29(1)$ & $8(1)$ & $5(1)$ & $1(1)$ \\
\hline C (25) & $43(1)$ & $32(1)$ & $39(1)$ & $3(1)$ & $12(1)$ & $-2(1)$ \\
\hline$C(26)$ & $48(1)$ & $31(1)$ & $46(1)$ & $9(1)$ & $3(1)$ & $-5(1)$ \\
\hline C (27) & $41(1)$ & $46(1)$ & $47(1)$ & $23(1)$ & $6(1)$ & $-5(1)$ \\
\hline C (28) & $52(1)$ & $54(1)$ & $49(1)$ & $20(1)$ & $26(1)$ & $3(1)$ \\
\hline C (29) & $48(1)$ & $38(1)$ & $41(1)$ & $9(1)$ & $19(1)$ & $4(1)$ \\
\hline
\end{tabular}

The anisotropic displacement factor exponent takes the form: $-2 \mathrm{pi}^{\wedge} 2\left[\mathrm{~h}^{\wedge} 2 \mathrm{a}^{\star \wedge} 2 \mathrm{U} 11+\ldots+2 \mathrm{~h} k \mathrm{a}^{\star} \mathrm{b}^{\star} \mathrm{U} 12\right.$ ]

Table 5. Hydrogen coordinates ( $x$ 10^4), isotropic displacement parameters (A^2 x 10^3), and occupancies for Compound 12 .

$\begin{array}{lllll}\text { x } & \text { y } & \text { U (eq) } & \text { Occ. }\end{array}$




\begin{tabular}{|c|c|c|c|c|c|}
\hline $\mathrm{H}(1 \mathrm{~A})$ & $-90(15)$ & $5323(13)$ & $1101(13)$ & $19(3)$ & 1 \\
\hline $\mathrm{H}(3 \mathrm{~A})$ & $3310(20)$ & $5183(18)$ & 3982 (17) & $51(5)$ & 1 \\
\hline $\mathrm{H}(3 \mathrm{~B})$ & $1620(20)$ & $5048(19)$ & 4205 (18) & $57(6)$ & 1 \\
\hline $\mathrm{H}(3 \mathrm{C})$ & $1830(20)$ & $4021(18)$ & $3044(16)$ & $42(4)$ & 1 \\
\hline $\mathrm{H}(6 \mathrm{~A})$ & $-1156(18)$ & $7228(15)$ & $2296(14)$ & $29(4)$ & 1 \\
\hline $\mathrm{H}(10 \mathrm{~A})$ & $-2550(30)$ & $8910(30)$ & $-810(20)$ & $89(8)$ & 1 \\
\hline $\mathrm{H}(10 \mathrm{~B})$ & $-860(40)$ & $9040(40)$ & $-720(30)$ & 147 (15) & 1 \\
\hline $\mathrm{H}(10 \mathrm{C})$ & $-1930(30)$ & $7700(20)$ & $-1390(20)$ & $80(7)$ & 1 \\
\hline $\mathrm{H}(12 \mathrm{~A})$ & 3654 (19) & $9512(16)$ & $4841(15)$ & $34(4)$ & 1 \\
\hline $\mathrm{H}(13 \mathrm{~A})$ & $4010(20)$ & 11749 (17) & $5838(16)$ & $45(5)$ & 1 \\
\hline $\mathrm{H}(14 \mathrm{~A})$ & $1960(20)$ & $12780(20)$ & $5359(18)$ & $56(5)$ & 1 \\
\hline $\mathrm{H}(15 \AA)$ & $-440(20)$ & $11828(19)$ & 3810 (17) & $54(5)$ & 1 \\
\hline $\mathrm{H}(16 \mathrm{~A})$ & $-760(20)$ & 9605 (18) & 2764 (17) & $47(5)$ & 1 \\
\hline $\mathrm{H}(20 \mathrm{~A})$ & $6100(30)$ & $7130(30)$ & $1710(20)$ & $90(8)$ & 1 \\
\hline $\mathrm{H}(20 \mathrm{~B})$ & $5440(20)$ & $5600(20)$ & $1358(18)$ & $58(6)$ & 1 \\
\hline $\mathrm{H}(20 \mathrm{C})$ & $4560(30)$ & $6420(20)$ & $400(20)$ & $88(8)$ & 1 \\
\hline $\mathrm{H}(23 \mathrm{~A})$ & $-1348(18)$ & $3382(15)$ & $1180(15)$ & $30(4)$ & 1 \\
\hline $\mathrm{H}(25 \mathrm{~A})$ & $-2510(20)$ & $1156(18)$ & $734(17)$ & $49(5)$ & 1 \\
\hline $\mathrm{H}(26 \mathrm{~A})$ & $-4160(20)$ & $-580(20)$ & $1110(18)$ & $61(6)$ & 1 \\
\hline $\mathrm{H}(27 \mathrm{~A})$ & $-5570(20)$ & $-200(20)$ & $2613(18)$ & $59(5)$ & 1 \\
\hline $\mathrm{H}(28 \mathrm{~A})$ & $-5090(20)$ & 2009 (19) & $3693(18)$ & $53(5)$ & 1 \\
\hline $\mathrm{H}(29 \mathrm{~A})$ & $-3420(20)$ & $3685(18)$ & $3416(16)$ & $44(5)$ & 1 \\
\hline
\end{tabular}

